EPJ Web of Conferences 20, 01008 (2012)

DOI: $10.1051 /$ epjconf/20122001008

() Owned by the authors, published by EDP Sciences, 2012

\title{
Spin structures of the pion and nucleon
}

\author{
Hyun-Chul Kim ${ }^{1, \mathrm{a}}$, Tim Ledwig ${ }^{2, \mathrm{~b}}$, and Seung-il $\mathrm{Nam}^{3, \mathrm{c}}$ \\ ${ }^{1}$ Department of Physics, Inha University, Incheon 402-751, Republic of Korea \\ ${ }^{2}$ Institut für Kernphysik, Universität Mainz, D-55099 Mainz, Germany \\ ${ }^{3}$ School of Physics, Korea Institute for Advanced Study (KIAS), Seoul 130-722, Republic of Korea
}

\begin{abstract}
We present recent studies on the transverse spin densities of the pion and nucleon within the framework of the chiral quark-(soliton) model, based on the calculation of the electromagnetic and tensor form factors of the pion and the nucleon. The results for the transverse spin densities of the quark inside a pion are in good agreement with the recent lattice data, while those of the nucleon show similar features to the lattice results. We also present the first results of the transverse spin densities of the strange quark inside a nucleon.
\end{abstract}

\section{Introduction}

The transversity of hadrons unveils a novel structure of quarks inside hadrons. Recently, the transverse spin asymmetry $A_{T T}$ of Drell-Yan processes in $p \bar{p}$ reactions provides valuable information on the transversity of the nucleon $[1,3,2,4]$. The transversity distribution $\delta q(x)$ was extracted [5] from the data of the azimuthal single spin asymmetry in SIDIS processes $l p^{\uparrow} \rightarrow l \pi X$, based on the Belle [6], HERMES [8,7] and COMPASS [9] experiments. In addition, the QCDSF/UKQCD Collaborations has carried out the first lattice calculation of the transverse spin structure of the nucleon [10].

Though the transversity of the pion is even less known than that of the nucleon, it has been studied also by the QCDSF/UKQCD Collaborations [11], combining the electromagnetic form factor of the pion with its tensor form factor. It was shown that when the quarks inside a pion are transversely polarized, their spatial distribution is strongly distorted. Motivated by the lattice work, Broniowski et al. [12] have studied the tensor form factor of the pion within the local and nonlocal Nambu-JonaLasinio (NJL) model. In order to compare their results with the lattice ones, they used a larger value of the pion mass, i.e. $m_{\pi}=600 \mathrm{MeV}$ such that the results can be confronted with the lattice data. They also considered the case of the chiral limit.

In the present talk, we want to review recent investigations $[13,14]$ on the transverse spin structures of the pion and the nucleon, based on the chiral quark-(soliton) model. The model is derived from the instanton vacuum that realizes the spontaneous chiral symmetry breaking $(\mathrm{S} \chi \mathrm{SB})$ naturally via quark zero modes. It may provide a good framework to study properties of the pion and the nucleon on the same footing. Note that there are only two parameters, that is, the average (anti)instanton size $\bar{\rho} \approx 1 / 3 \mathrm{fm}$ and average inter-instanton distance $\bar{R} \approx 1 \mathrm{fm}$. The normalization point is determined by the average size of instantons and is almost equal to $\rho^{-1} \approx 600-700 \mathrm{MeV}$. The values of the $\bar{\rho}$ and $\bar{R}$ were estimated many years ago phenomenologically in Ref. [15] as well as theoretically in Ref. [16-18]. The instanton framework has been proved to be reliable in reproducing experimental data especially for the meson sector, such as the meson distribution amplitudes [19-21], semileptonic decays [22], and etc. In the case of the nucleon, we will switch off the momentum dependence of the

\footnotetext{
a e-mail: hckim@inha.ac.kr

b e-mail: ledwig@kph.uni-mainz.de

c e-mail: sinam@kias.re.kr
}

This is an Open Access article distributed under the terms of the Creative Commons Attribution-Noncommercial License 3.0, which permits unrestricted use, distribution, and reproduction in any noncommercial medium, provided the original work is properly cited. 
dynamical quark mass for convenience and take it as a free parameter. However, we will employ the value of $420 \mathrm{MeV}$ with which almost all properties of the nucleon and hyperons were successfully described [23].

\section{Generalized form factors of the pion and nucleon}

The probability densities of transversely polarized quarks for the $n$th moment and the first moment of the pion and the nucleon are respectively expressed as

$$
\begin{aligned}
\rho_{n}\left(b_{\perp}, s_{\perp}\right)= & \frac{1}{2}\left[A_{n 0}\left(b_{\perp}^{2}\right)-\frac{s_{\perp}^{i} \epsilon^{i j} b_{\perp}^{j}}{m_{\pi}} \frac{\partial B_{n 0}\left(b_{\perp}^{2}\right)}{\partial b_{\perp}^{2}}\right], \\
\rho\left(b_{\perp}, S_{\perp}, s_{\perp}\right)= & \frac{1}{2}\left[H\left(b^{2}\right)-S^{i} \epsilon^{i j} b^{j} \frac{1}{M_{N}} \frac{\partial E\left(b^{2}\right)}{\partial b^{2}}-s^{i} \epsilon^{i j} b^{j} \frac{1}{M_{N}} \frac{\partial \kappa_{T}\left(b^{2}\right)}{\partial b^{2}}\right. \\
& \left.+s^{i} S^{i}\left\{H_{T}\left(b^{2}\right)-\frac{1}{4 M_{N}^{2}} \nabla^{2} \tilde{H}_{T}\left(b^{2}\right)\right\}+s^{i}\left(2 b^{i} b^{j}-b^{2} \delta^{i j}\right) S^{j} \frac{1}{M_{N}^{2}}\left(\frac{\partial}{\partial b^{2}}\right)^{2} \tilde{H}_{T}\left(b^{2}\right)\right],
\end{aligned}
$$

where $b_{\perp}$ denote the impact parameter that measures the distance from the center of momentum of the pion to the quark in the transverse plane to its motion. The $s_{\perp}$ stands for the fixed transverse spin of the quark. For simplicity, we choose the $z$ direction for the quark longitudinal momentum. The $x$ indicates the momentum fraction possessed by the quark inside the pion. The tensor $\epsilon^{i j}$ is an antisymmetric tensor with the property $\epsilon^{12}=-\epsilon^{21}=1$. The operator $\nabla^{2}$ is a Laplacian with respect to $\boldsymbol{b}$. The Fourier transform of the form factors are defined generically as

$$
F\left(b_{\perp}^{2}\right)=\frac{1}{(2 \pi)^{2}} \int d^{2} q_{\perp} e^{-i b_{\perp} \cdot q_{\perp}} F\left(q_{\perp}^{2}\right)=\frac{1}{2 \pi} \int_{0}^{\infty} \operatorname{QdQJ}_{0}(b Q) F\left(Q^{2}\right),
$$

$F\left(b_{\perp}^{2}\right)$ designates the generic form factors appearing in Eq.(1). The magnitudes of the transverse momentum and impact parameter are expressed as $\left|\boldsymbol{q}_{\perp}\right| \equiv Q$ and $\left|\boldsymbol{b}_{\perp}\right| \equiv b$. Similarly, the Fourier transform of the derivative of the GFF with respect to $b_{\perp}^{2}$ can be evaluated as:

$$
\frac{\partial F\left(b_{\perp}^{2}\right)}{\partial b_{\perp}^{2}} \equiv F^{\prime}\left(b_{\perp}^{2}\right)=-\frac{1}{4 \pi b} \int_{0}^{\infty} Q^{2} d Q J_{1}(b Q) F\left(Q^{2}\right) .
$$

The $J_{0}$ and $J_{1}$ in Eqs. $(2,3)$ denote the Bessel functions of order 0 and 1, respectively.

For the first moment, the GFFs $A_{10}$ and $B_{10}$ are identified with the electromagnetic and tensor form factors of the pion, respectively. Previously, we have studied $A_{10}\left(q^{2}\right)$ in the momentum space within the nonlocal chiral quark model (NL $\chi \mathrm{QM})$ from the instanton vacuum [24], resulting in a good agreement with the experimental data. The $H$ and $E$ correspond to the Dirac and Pauli form factors of the nucleon [14]. The $H_{T}$ and $\kappa_{T}$ denotes tensor and tensor anomalous form factors, respectively. Specific spin directions of the quark and the nucleon being selected, the last term with $\tilde{H}_{T}$ in Eq.(1) does not contribute to the transverse spin densities.

\section{Results and discussion}

Figure 1 shows respectively the numerical results for the electromagnetic form factor of the pion $A_{10}$ in the left panel and its tensor form factor $B_{10}$ in the right panel as functions of $Q^{2}$ in the range of $0 \leq Q^{2} \leq 1 \mathrm{GeV}^{2}$. The results for the electromagnetic form factor of the pion were already shown to be in good agreement with the experimental data [24]. As for the generalized tensor form factors of the pion, there is no experimental data available to date. Thus, we compare the present results with the ones from the recent lattice calculation and find that they are in excellent agreement with each other.

In Fig. 2, we draw the results of the flavor-decomposed tensor and tensor anomalous form factors in the left and right panels, respectively, in comparison with the lattice data. While the values of the 

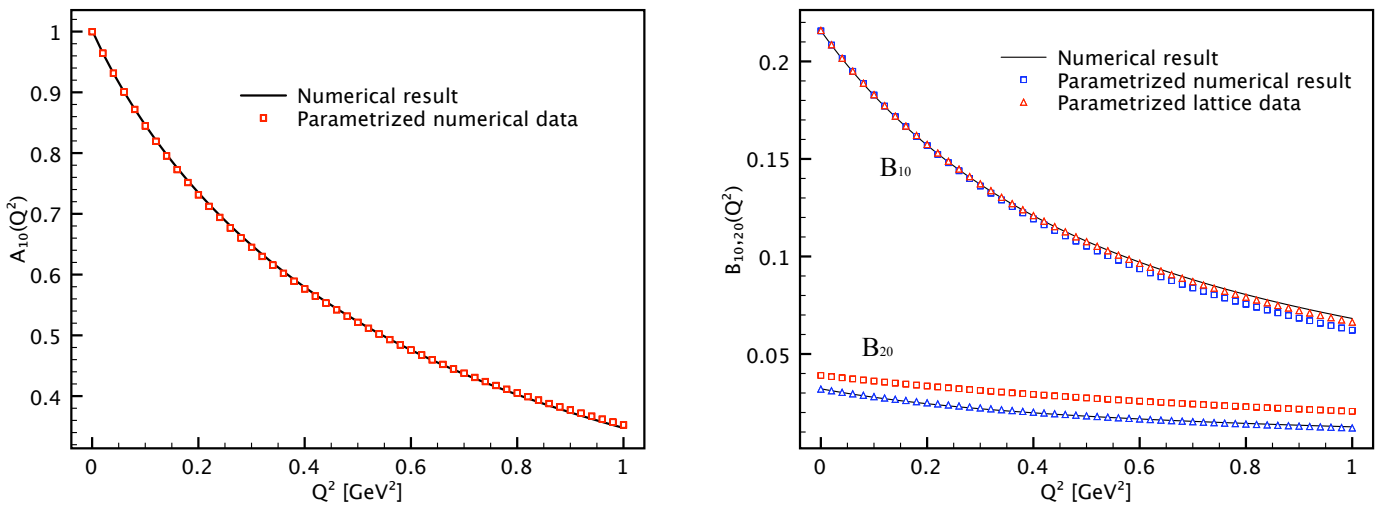

Fig. 1. The numerical results for the electromagnetic form factor $A_{10}\left(Q^{2}\right)$ are presented in the left panel and those for the tensor form factors $B_{10}\left(Q^{2}\right)$ and $B_{20}\left(Q^{2}\right)$ in the right panel. The solid curves depict the numerical results, whereas their parametrizations are denoted by the squares [13]. We also show the parametrized lattice data [11] for the tensor form factors, designated by the triangles in the right panel.
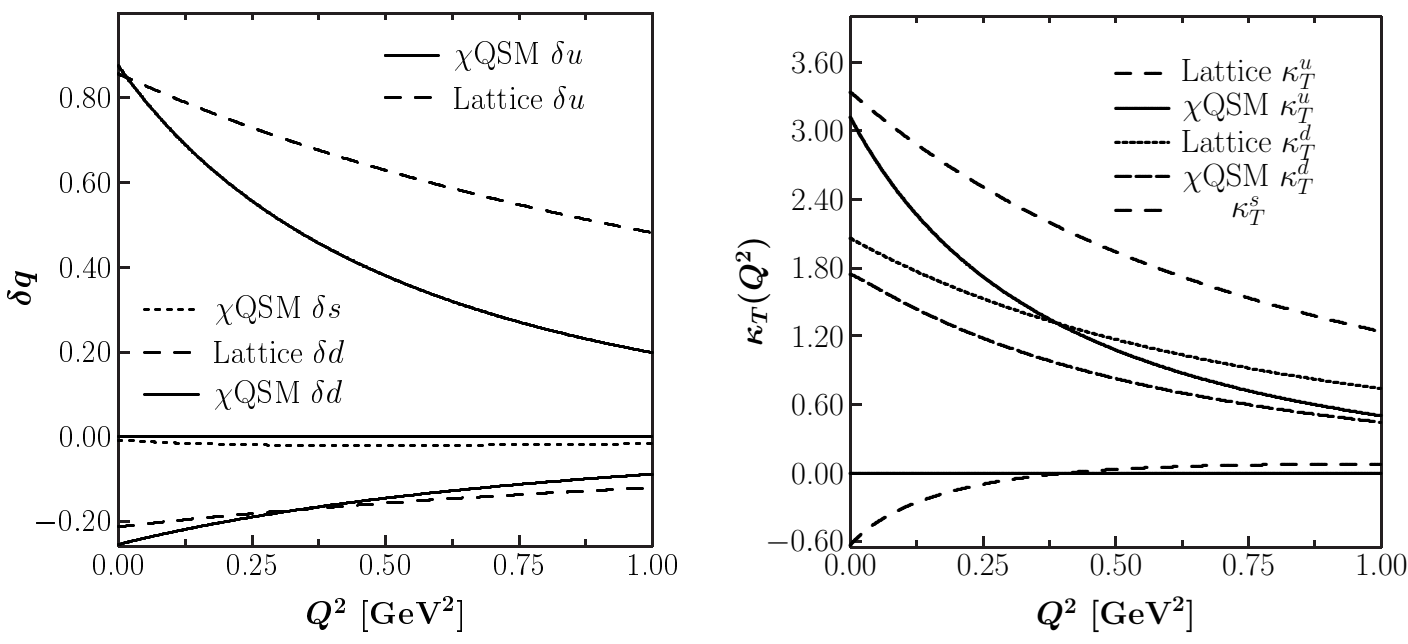

Fig. 2. The numerical results for the electromagnetic form factor $A_{10}\left(Q^{2}\right)$ are presented in the left panel and those for the tensor form factors $B_{10}\left(Q^{2}\right)$ and $B_{20}\left(Q^{2}\right)$ in the right panel. The solid curves depict the numerical results, whereas their parametrizations denoted by the squares [14]. We also show the parametrized lattice data [11] for the tensor form factors, designated by the triangles in the right panel.

tensor charges and anomalous tensor magnetic moment are comparable with the lattice results, the $Q^{2}$ dependences seem different.

Putting the results of the densities obtained from the Fourier transform of the form factors shown in Fig. 2, we derive the transverse quark densities of the pion as shown in Fig. 3. As expected from Eq.(1), the unpolarized quarks are distributed symmetrically on the $b_{x}-b_{y}$ plane. On the other hand, the spatial distribution of a transversely polarized quark inside the pion are distorted as shown in the right panel of Fig. 3. This is soley due to the finite values of the pion tensor form factor.

In the case of the nucleon, we can see a richer transverse spin structure. This is natural, because the nucleon has spin $1 / 2$ and consists of three valence quarks and even of strange quarks arising from the Dirac-sea polarization. Figure 4 depict the transverse up and down quark spin densities inside a nucleon. In the left panel,the density of unpolarized up and down quarks are drawn in a transversely polarized nucleon for $[S, s]=[(1,0),(0,0)]$. We note from Eq.(1) that the distortion of these densities are mainly governed by the Pauli form factors. We see that the down quark density is more distorted in 

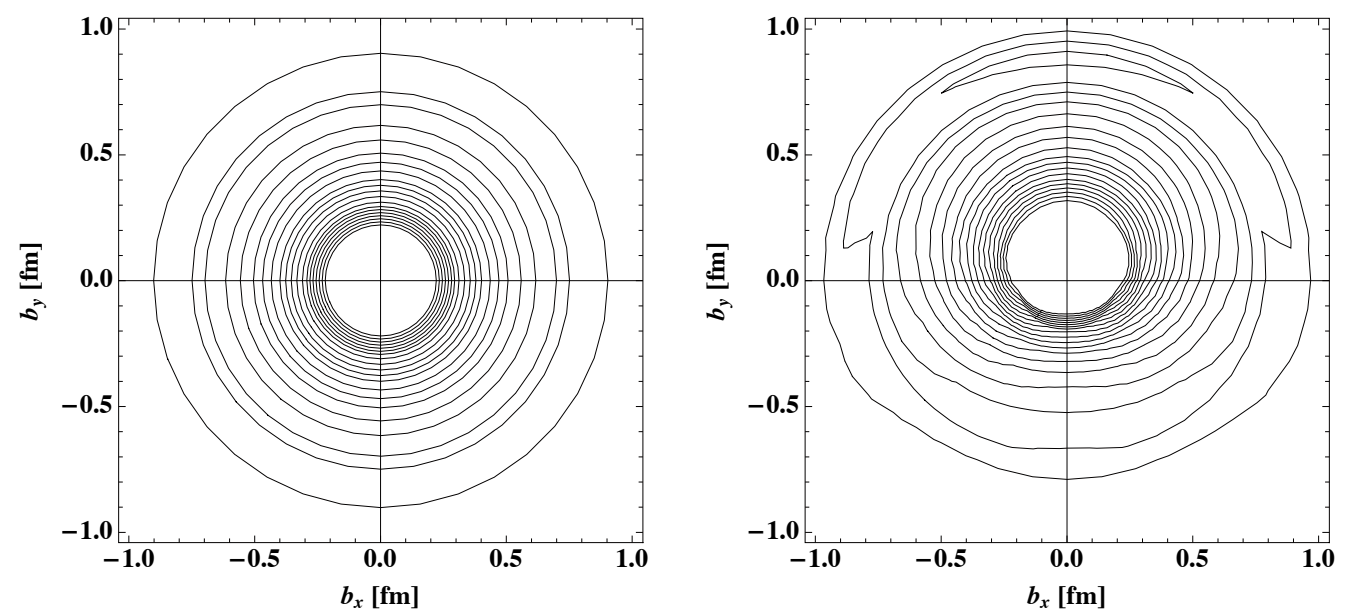

Fig. 3. Unpolarized (left) and polarized (right) probability densities, $\rho_{1}$ in Eq. (1) as a function of $b_{x}$ and $b_{y}$ for $s_{\perp}=(+1,0)$.

the negative direction of $b_{y}$ than the up quark density in the positive direction. This can be understood from the fact that the down Pauli form factor falls off more slowly than that of the up quark. Moreover, the down anomalous magnetic moment $\left(\kappa^{d}=-1.80\right)$ is known to be negative. The sign of the form factors at intermediate $Q^{2}$ determines the direction of the shift. If we choose the polarizations as $[S, s]=[(0,0),(1,0)]$, only the anomalous tensor magnetic form factors enter, as shown in the right panel of Fig. 4. Both $\kappa_{T}^{u}$ and $\kappa_{T}^{d}$ are positive, so that both transverse spin densities are deformed in the direction of positive $b_{y}$ and again. Since the form factor $\kappa_{T}^{d}$ falls off more slowly, the density for the down quark is more strongly deformed.

Both the strange Pauli form factor and anomalous tensor magnetic form factor turn out to be rather small, so is the strength of the strange densities. In Fig. 5 we draw the transverse strange quark spin densities with the lowest moment. Note that the magnitudes of the densities are smaller than those of the up and down quarks by an order of magnitude. As shown in the right panel, the density of transversely polarized quarks in a unpolarized nucleon is strongly distorted. It is due to the $Q^{2}$ dependence of $\kappa_{T}^{s}\left(Q^{2}\right)$ as shown in Fig. 2. Moreover, the density becomes negative for the negative values of $b_{y}$. Moreover, the density of unpolarized strange quarks in a polarized nucleon is negatively shifted. The reason can be found in the fact that the strange Pauli form factor $F_{2}^{s}$ turns negative from $Q^{2} \approx 0.2 \mathrm{GeV}^{2}$ and the lower $Q^{2}$ values are suppressed in the Fourier transform. Thus, the negative values of the form factor at intermediate $Q^{2}$ make the density shifted to the negative $b_{y}$ direction, despite the positive strange anomalous magnetic moment.

\section{Summary and conclusion}

In the present talk, we reported recent investigations on the transverse quark spin structures of the pion and the nucleon, based on the chiral quark-(soliton) model. The results for the pion are in good agreement with the lattice data, while those for the nucleon are qualitatively in agreement with the lattice ones. We also showed the first results of the transverse strange quark densities inside a nucleon. For details, we refer to Refs. [13,14].

\section{Acknowledgments}

The present work was supported by Basic Science Research Program through the National Research Foundation of Korea (NRF) funded by the Ministry of Education, Science and Technology (grant number: 2010-0016265). 

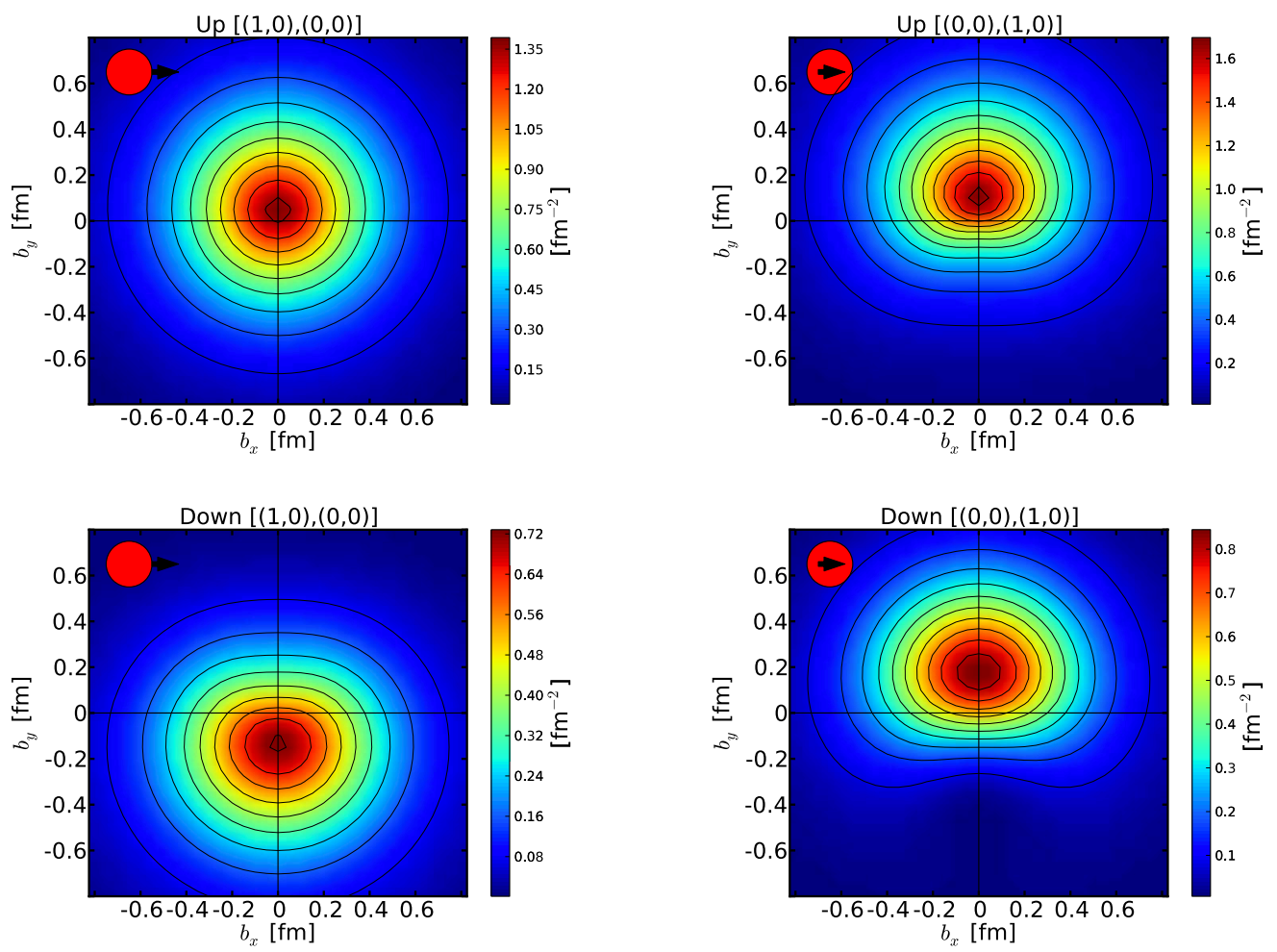

Fig. 4. Transverse up and down quark spin densities with the lowest moment of the nucleon from the $\chi \mathrm{QSM}$. In the upper left panel, the density of unpolarized quarks in a transversely polarized nucleon $([\boldsymbol{S}, \boldsymbol{s}]=[(1,0),(0,0)])$ is drawn and in the upper right panel, that of transversely polarized quarks in a unpolarized nucleon $([S, s]=$ $[(0,0),(1,0)])$. In the lower panel, we plot the down quark densities.

\section{References}

1. A.V. Efremov, K. Goeke and P. Schweitzer, Eur. Phys. J. C 35 (2004) 207.

2. V. Barone, [PAX Collaboration], hep-ex/0505054.

3. M. Anselmino, V. Barone, A. Drago and N.N. Nikolaev, Phys. Lett. B 594 (2004) 97.

4. B. Pasquini, M. Pincetti and S. Boffi, Phys. Rev. D 76 (2007) 034020.

5. M. Anselmino et al., Nucl. Phys. Proc. Suppl. 191 (2009) 98.

6. K. Abe et al. [Belle Colaboration], Phys. Rev. Lett. 96 (2006) 232002.

7. L. Pappalardo for the HERMES collaboration, Apr 2006. 4pp. Prepared for 14th International Workshop on Deep Inelastic Scattering (DIS 2006), Tsukuba, Japan, 20-24 Apr 2006.

8. A. Airapetian et al., [HERMES Collaboration], Phys. Rev. Lett. 94 (2005) 012002.

9. E.S. Ageev et al., [COMPASS Collaboration], Nucl. Phys. B 765 (2007) 31.

10. M. Göckeler et al. [QCDSF Collaboration and UKQCD Collaboration], Phys. Rev. Lett. 98 (2007) 222001.

11. D. Brommel et al. [QCDSF/UKQCD Collaboration], Phys. Rev. Lett. 101 (2008) 122001.

12. W. Broniowski, A. E. Dorokhov and E. R. Arriola, Phys. Rev. D 82, 094001 (2010).

13. S. i. Nam and H.-Ch. Kim, Phys. Lett. B 700 (2011) 305 [arXiv:1010.0468 [hep-ph]].

14. T. Ledwig and H.-Ch. Kim, arXiv:1107.4952 [hep-ph].

15. E. V. Shuryak, Nucl. Phys. B 203 (1982) 93.

16. D. Diakonov and V. Y. Petrov, Nucl. Phys. B 245 (1984) 259.

17. D. Diakonov, Prog. Part. Nucl. Phys. 51 (2003) 173.

18. T. Schäfer and E. V. Shuryak, Rev. Mod. Phys. 70 (1998) 323. 

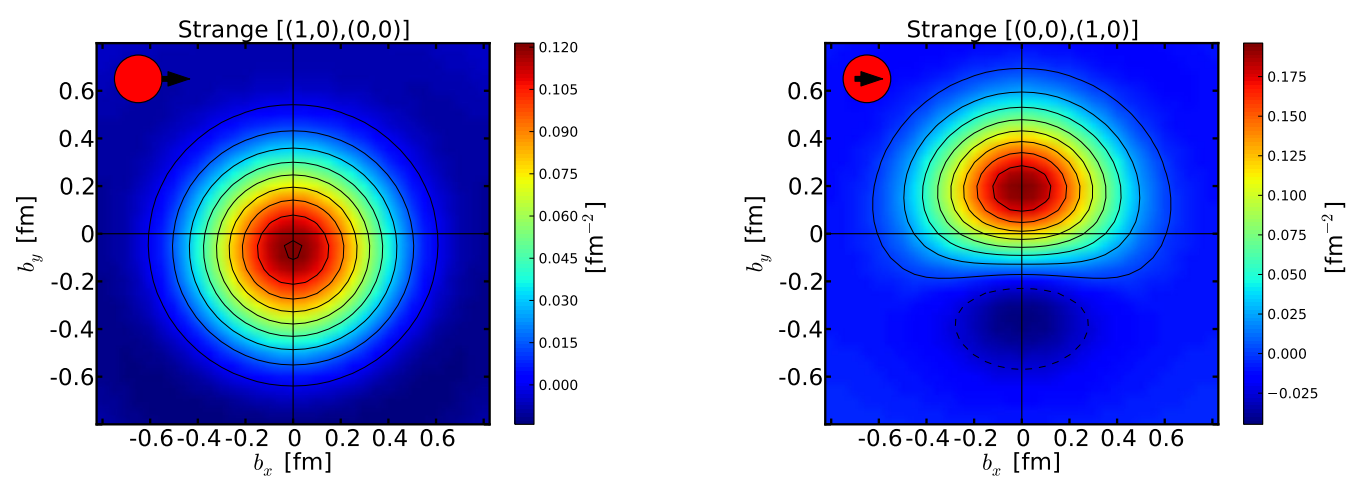

Fig. 5. Transverse strange quark spin densities with the lowest moment of the nucleon from the $\chi \mathrm{QSM}$. In the left panel, the density of unpolarized quarks in a transversely polarized nucleon $([\boldsymbol{S}, \boldsymbol{s}]=[(1,0),(0,0)])$ is drawn and in the right panel, that of transversely polarized quarks in a unpolarized nucleon $([\boldsymbol{S}, \boldsymbol{s}]=[(0,0),(1,0)])$.

19. S. i. Nam and H. -Ch. Kim, Phys. Rev. D 74 (2006) 076005.

20. S. i. Nam and H. -Ch. Kim, Phys. Rev. D 74 (2006) 096007.

21. S. i. Nam, H. -Ch. Kim, A. Hosaka and M. M. Musakhanov, Phys. Rev. D 74 (2006) 014019.

22. S. i. Nam and H. -Ch. Kim, Phys. Rev. D 75 (2007) 094011.

23. C.V. Christov et al., Prog. Part. Nucl. Phys. 37 (1996) 91.

24. S. i. Nam and H. -Ch. Kim, Phys. Rev. D 77 (2008) 094014.

25. A. Silva, Ph.D. Dissertation, Ruhr-Universität Bochum, (unpublished).

26. A. Silva, H. -Ch. Kim and K. Goeke, Phys. Rev. D65 (2002) 014016.

27. A. Silva, H. -Ch. Kim and K. Goeke, Eur. Phys. J. A22 (2004) 481.

28. K. Goeke, H.-Ch. Kim, A. Silva and D. Urbano, Eur. Phys. J. A32 (2007) 393.

29. T. Ledwig, A. Silva and H.-Ch. Kim, Phys. Rev. D 82 (2010) 034022.

30. T. Ledwig, A. Silva and H.-Ch. Kim, Phys. Rev. D 82 (2010) 054014.

31. C. C. Carlson and M. Vanderhaeghen, Phys. Rev. Lett. 100 (2008) 032004; Eur. Phys. J. A 41 (2009) 41.

32. L. Tiator and M. Vanderhaeghen, Phys. Lett. B 672 (2009) 344.

33. C. Lorce, B. Pasquini, M. Vanderhaeghen, arXiv:1102.4702

34. C. Lorce, B. Pasquini, arXiv:1106.0139

35. Ph. Hägler et al., Phys. Rev. D 68 (2003) 034505. 